\title{
Electric mine motor thermal models aiding design and setting thermal protections
}

\author{
R. $\mathrm{KROK}^{*}$ \\ Institute of Electrical Engineering and Informatics, Division of Electrical Machines and Electrical Engineering in Transport, \\ Silesian University of Technology, 10A Akademicka St., 44-100 Gliwice, Poland
}

\begin{abstract}
The paper presents original modified thermal networks for calculations of the temperature field in induction mine motors under steady and transient conditions aiding design and setting thermal protections. They can be used for location of the hottest places in motor elements in order to place in them sensors for temperature measurements. They can be also applied to determining the equivalent thermal constants of windings needed when setting microprocessor thermal protections.
\end{abstract}

Key words: induction mine motors, thermal protections, thermal networks.

\section{Introduction}

Induction mine motors working under very difficult environmental conditions in coal mine underground are particularly exposed to damages. Since they are high power motors, their repair costs are really high. Hence, it is especially essential to apply efficient protection systems of mine motors.

From experts' reports concerning mine motors it follows that their thermal damages caused by exceeding the permissible temperature of construction elements, especially the stator winding insulation system, occur very often. The microprocessor protections of large electric motors used nowadays can cooperate with sensors measuring temperature in construction elements exposed to thermal damages. A very essential issue is selecting a proper place for install sensors because they should measure the maximum temperatures of elements. In particular parts of a mine motor there occur significant non-uniformities of the temperature distribution due to large overall dimensions as well as relatively long cooling ducts through which air or water flows. The difference between the maximum and minimum temperature, for instance in the motor stator winding, equals even tens of K. In order to locate the places with the maximum temperature in motor construction elements, it is required to create thermal models enabling calculations of the temperature field in them. It is not enough to calculate the average temperature.

Determining settings for microprocessor protections a mine induction motor requires not only the knowledge of its ratings (rated current, rated voltage and so on), but also some technical parameters which are not given, as a rule, by manufacturers. The determination of values of the equivalent thermal time constants for heating the motor windings makes many difficulties. In case of large air-cooled induction motors (both for close and open housing) used besides the coal-mining industry, one can find in literature the guidelines concerning the way of assessing the values of the equivalent thermal time constants for heating the motor windings for the purpose of setting the thermal protections $[1,2]$. However, such simplified methods for assessing the values of those constants for air cooled mine motors do not exist. Manufacturers of mine motors use many different solutions of the water cooling system for: frame, bearing discs, and sometimes shaft. In some types of construction an additional, inner circulation of cooling air is used. Onespeed motors have one winding, while twospeed ones have two independent or one switchable winding in the stator. These main differences in construction of various mine motors make elaborating universal, simplified relationships enabling calculations of thermal time constants for heating the windings impossible. Therefore, design and setting protections require developing thermal models of mine motors of different construction and different cooling system in order to calculate the temperature field in transient thermal states. It is possible to determine the equivalent thermal time constants for heating the windings basing on the results of motor heating simulations.

\section{Thermal networks in calculations of the temperature distribution in electric machines}

The thermal network method, belongs to the group of the oldest methods used for calculations of the temperature distribution in electric motors and transformers. First equivalent thermal diagrams of electromagnetic objects contained several nodes only [3]. In consequence, they enabled calculation of approximated values of the average temperatures of several selected elements of a machine. The small number of the thermal diagram nodes resulted from necessity of its analytical solution.

The use of analog and, next, digital machines for solving thermal networks enabled significant increase in the number of nodes [4-12]. When creating these models the simplifi-

*e-mail: Roman.Krok@polsl.pl 
cation was used which relies on assuming that the temperature distribution in streams of the cooling media flowing in ducts was linear along their length. There was often adopted a greater simplification consisting in assuming the constant temperature of the medium flowing in a duct along its whole length.

Nowadays the available programs installed on computers of typical calculating powers solve thermal networks containing even several thousand nodes in a very short time. It has enabled modelling two-, even three-dimensional temperature distributions in electric machines when taking into account nonlinear temperature distributions in medium streams flowing through cooling ducts as well as heat accumulation occurring in them in transient thermal states [13, 14].

The basic problem when creating thermal networks for large ac machines (including mine motors) is modelling the heating of streams of water or gas flowing in very extended networks of cooling ducts. Additionally, in elements of these machines there occur non-uniformities of the temperature distribution caused by their large overall dimensions and significant heating of the cooling medium streams by the heat carried away from the duct surfaces. As a result, the maximal temperature differences inside the elements reach the value of a dozen or so, sometimes even dozens of K. The average temperatures of the mine motor elements calculated with use of the classical thermal networks are not sufficient when designing protection systems as well as determining their settings. The thermal model has to represent the temperature distributions inside particular machine parts and in cooling medium streams both under steady and transient thermal conditions.

The original modified thermal networks for mine motors created by the author enable determination of the temperature distribution inside all construction elements of the motor as well as in the streams of water and air flowing in cooling ducts. The thermal network nodes represent the average temperatures of difference elements into which particular motor parts were divided.

Modification of thermal networks consists in not making the following simplifications:

- short-circuit of the nodes representing the cooling medium temperature in sections of the ducts (one node represents the average temperature of medium in the whole cooling duct),

- assuming the linear temperature distribution in the cooling medium stream along the duct length (consequence of simplification consisting in assuming constant density of the heat stream passing from the cooling duct surface into medium along its whole length).

Additionally, in the modified thermal networks there were introduced thermal capacitances of the cooling medium flowing in separated difference elements of the duct in order to calculate transient thermal states. The new equivalent thermal diagram for a medium stream flowing in a cooling duct divided into difference elements developed by the author [13, 14] represents the nonlinear temperature distributions in the streams of water and air flowing in cooling ducts of mine motors under steady and transient thermal conditions. It enables the joint modelling of phenomena occurring in the motor construction elements and streams of cooling media flowing in them when taking into account an interaction between them. In a consequence, the thermal network elaborated for mine motors represents temperature distributions in media flowing in extended networks of cooling ducts as well as the resulting non-uniformities of the temperature distribution in cooled elements. Such a way of modelling the temperature field in large ac machines has not been used, so far.

The temperature distribution calculated in a mine motor with use of the modified thermal network is close to the continuous distribution obtained as a result of solving a system of partial differential equations of thermal conduction. The motor consists of a large number of elements connected with each other, made of materials of different thermal properties, inside which the cooling medium flows. The temperature field in such a type of object is described by a system of partial differential equations of thermal conduction formulated for particular construction elements and cooling media streams. After transformations, the derived system of equations can be brought to a partial differential equation of high order. Analytical formulas for roots were derived for the characteristic equation of fifth order at most. Due to a large number of the motor elements connected with each other, the order of the characteristic equation is always much higher than five. As a result, its roots should be determined in an approximate way by numerical methods. So, the analytical solving of a system of differential equations of thermal conduction is very timeconsuming and results in obtaining only approximate temperature distributions in motor elements.

The high accuracy of calculating the temperature field by means of the modified thermal networks developed by the author was confirmed by measurements taken on many types of mine motors working under different load and different cooling conditions [13]. The dimension of the solved system of equations describing the temperature field in a motor is significantly smaller than that for the finite element method (FEM). In the process of creating the mesh by FEM, very thin and at the same time very long insulation layers as well as air gaps are divided into elements whose overall dimensions have to be of the same order as their thickness. The process of creating the mesh in very long cooling ducts of small diameter is similar. In many cases in practice it causes significant exceeding the permissible number of elements and nodes in the model (even when using programs installed in working stations) as well as, additionally, a very long time of computations. In publication [13] there are compared the thermal models of an exemplary deep-slot motor rotor made with use of the FEM and thermal network. The courses of the maximum temperature in the rotor cage bars at motor starting calculated by means of the thermal network containing only 32 nodes differ at most by $1.9 \mathrm{~K}$ from those calculated with use of the network containing 540 nodes made in program COSMOS/M using FEM.

The modified thermal networks of mine motors enable calculating the temperature field in motors together with streams 
of cooling water and air with high accuracy and during a significantly shorter time than when using FEM.

\section{Modified thermal networks for mine motors}

The modified thermal networks enable calculating the temperature field in one- and two-speed (with one switchable or two independent windings in the stator) induction mine motors of different construction in steady and transient thermal states.

In the considered motors the basic construction elements (frame, stator core, stator winding, rotor cage and so on) can be inscribed into thin-walled rings. Streams of cooling media (air or water) flow in ducts placed in the elements in the axial direction. The consequence of such motor construction is that the temperature inside elements changes significantly in the axial direction, while in the radial direction it is practically constant.

In order to create a modified thermal network for a mine motor it was necessary to separate construction elements inscribed into thin-walled rings. Those elements together with the cooling ducts placed in them were divided in the axial direction (in which there exists the largest nonuniformity of the temperature distribution) into slices $\Delta x$ wide - Fig. 1. In that way in particular elements there were separated the difference elements, and next to each of them one thermal node was assigned.

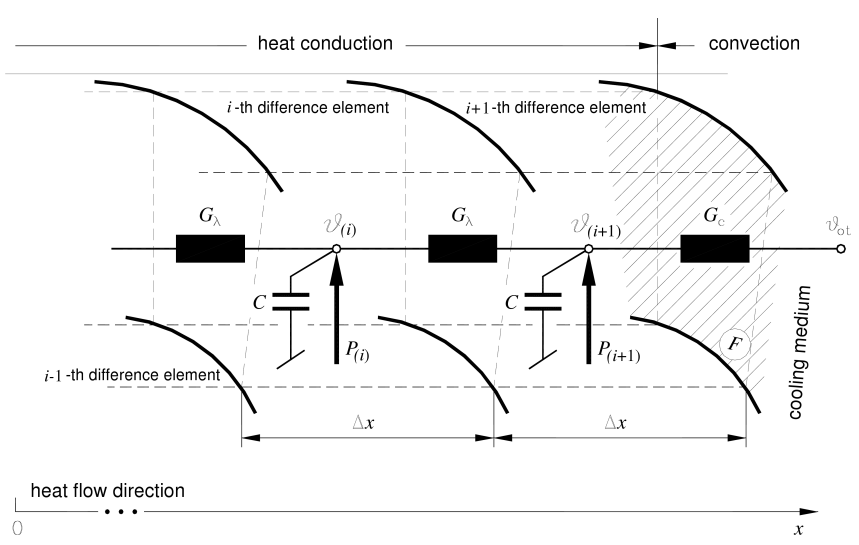

Fig. 1. Division of the exemplary motor construction part into elements in the axial direction and assigning the thermal network to it

The value of the parameter $\Delta x$ determining the numbers of division of particular elements into difference elements was determined for each motor separately by investigating the solution sensitivity (calculated temperature distributions in motor elements and cooling medium streams) to its changes. If the calculated temperature changes when decreasing the $\Delta x$ value do not exceed the assumed accuracy of numerical calculations (in the created thermal networks there was assumed the accuracy of numerical calculations equal to $0.1 \mathrm{~K}$ ), the numbers of division of the motor elements obtained as a result of discretization are recognized to be optimal. Then the optimal division of the motor into difference elements ensures obtaining the assumed accuracy of numerical calculations for the smallest possible number of the separated difference elements.
The equivalent conductivities for the heat streams flowing due to conduction between the neighbouring difference elements (Fig. 1) were determined from the relationship:

$$
G_{\lambda}=\frac{\lambda_{x} \cdot F}{\Delta x}
$$

where $\lambda_{x}$ - thermal conductivity of the material from which the constructional element in the $x$ axis direction is made of, $F$ - surface area of the wall separating the neighbouring difference elements.

The equivalent conductivities for the heat streams carried away from the walls of the difference elements (Fig. 1) to the cooling media flowing around them were determined from the relation:

$$
G_{c}=\alpha_{c} \cdot F
$$

where $\alpha_{c}$ - heat transfer coefficient, $F$ - surface area of the element wall, from which the heat is transferred to the cooling medium flowing around it.

The thermal capacity of the difference elements separated in the motor construction elements (Fig. 1) was determined from the relationship:

$$
C=c \cdot \gamma \cdot \Delta x \cdot F
$$

where $c-$ specific heat of the material per a unit mass, $\gamma-$ density of the material the element is made of.

In the difference elements separated inside the stator and rotor windings (Fig. 1) there occur power losses dependent on temperature:

$$
P_{(i)}=P_{0} \cdot\left(1+\alpha \cdot \vartheta_{(i)}\right), \quad P_{0}=k_{a} \cdot j^{2} \cdot \rho_{0} \cdot \Delta x \cdot F
$$

where $P_{0}$ - power losses in the separated difference elements in the reference temperature (assumed to be equal to $0^{\circ} \mathrm{C}$ ), $\alpha$ - temperature coefficient of resistance change of the material the windings are made of, $\vartheta_{(i)}$ - average temperature of the $i$-th difference element, $k_{a}$-additional loss coefficient, $j-$ average current density in the winding, $\rho_{0}$ - resistivity of the material the winding is made of in the reference temperature.

On the motor thermal diagram there are also power sources (Fig. 2) of discharges equal to the thermal powers convected by the streams of media flowing through the cooling ducts:

$$
P_{m(i)}=\stackrel{\bullet}{m} c_{p} \vartheta_{m(i)},
$$

where $\dot{m}$ - stream of cooling medium mass, $c_{p}$ - specific heat of the cooling medium per a unit mass at constant pressure (of water or air treated as an ideal gas), $\vartheta_{m(i)}$ - average medium temperature in the $i$-th difference element separated inside the duct. 


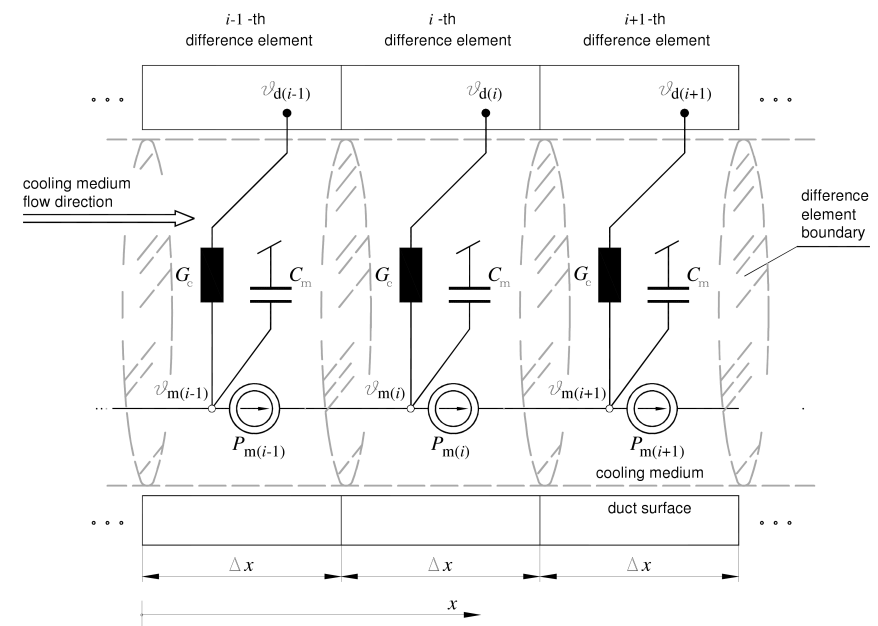

Fig. 2. Thermal network for a duct with flowing cooling medium stream

The thermal capacity of the cooling medium flowing through the duct difference elements (Fig. 2) was calculated from the formula:

$$
C_{m}=c_{p} \cdot \gamma_{m} \cdot \Delta x \cdot S,
$$

where $\gamma_{m}$ - medium density, $S$ - cross-sectional area surface of the duct.

\section{Thermal network for a two-speed mine motor}

In this section, there are presented the construction, cooling system and thermal network of mining motors with a water cooled frame on example of a $85 / 250 \mathrm{~kW}$ double speed induction motor. The motor under consideration has a helical water duct (Figs. 3 and 4) in the frame. Water flows between two concentric tubes. The partition plates welded to the inner tube force the water flow along a helical path. The water flows in the frame from the side of a clutch (C) and flows out at the opposite side $(\mathrm{O})$.

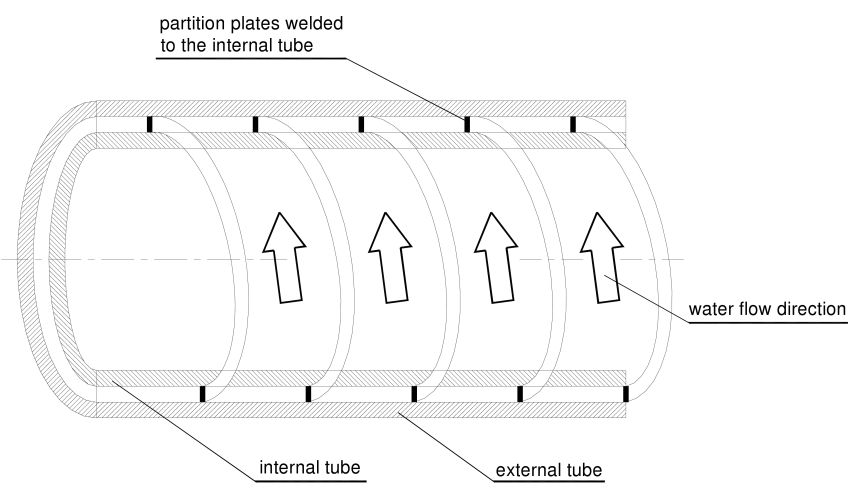

Fig. 3. Cooling water flow in a helical cooling duct in the mine motor frame

The inner air circulation in the motor (in Fig. 4 arrows denote directions of the air flow) is forced by a radial fan mounted on the shaft at the side of $(\mathrm{O})$. The air is heated when flowing through axial ducts in the rotor core and is cooled when flowing through axial ducts in a water cooler in the frame. The heat is carried away from the rotor active elements to the cooling water flowing in the frame by means of the air stream.

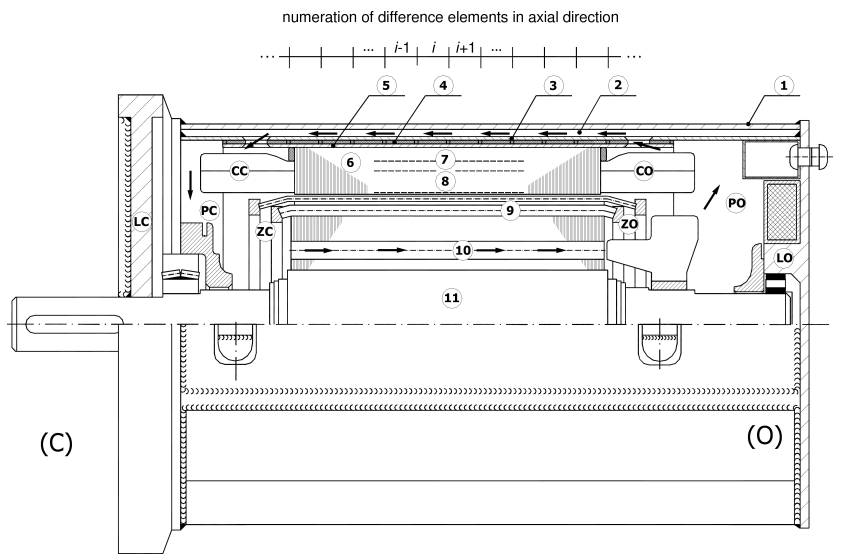

Fig. 4. Section of the $85 / 250 \mathrm{~kW}$ double speed mine motor with a water cooled frame

In the motor there were separated the construction elements taking part in the heat exchange (Fig. 4), and next they were denoted as follows:

- flame-proof enclosure (motor casing) - 1 ,

- axial air ventilation ducts in the frame -2 ,

- external tube (Fig. 3) in the water cooler in the frame - 3,

- helical water duct in the frame - 4,

- internal tube in the water cooler in the frame -5 ,

- stator core -6 ,

- slot part of the stator winding of a number of poles $2 p=4$ -7 ,

- slot part of the stator winding of a number of poles $2 p=12$ -8 ,

- bars of the rotor cage -9 ,

- axial air ventilation ducts in the rotor core -10 ,

- shaft together with rotor laminations - 11,

- stator head windings at the side $(\mathrm{C})$ and $(\mathrm{O})$ - CC and CO, respectively,

- rings shorting rotor cage bars at the side $(\mathrm{C})$ and $(\mathrm{O})-\mathrm{ZC}$ and $\mathrm{ZO}$, respectively,

- bearing discs at the side $(\mathrm{C})$ and $(\mathrm{O})$ - LC and LO, respectively,

- air in the surrounding of stator head windings and rings shorting rotor cage bars at the side $(\mathrm{C})$ and $(\mathrm{O})-\mathrm{PC}$ and $\mathrm{PO}$, respectively.

In the motor thermal network worked out (Fig. 5) there were taken into account:

- heat flow in particular constructional elements in axial and radial direction,

- different values of thermal conductivity of the stator and rotor cores in axial and radial direction,

- heat convection in water and air streams flowing through cooling ducts in the frame and rotor core,

- temperature changes in cooling media (water and air) streams along the duct length caused by the heat flow from the duct surface (or in opposite direction), 


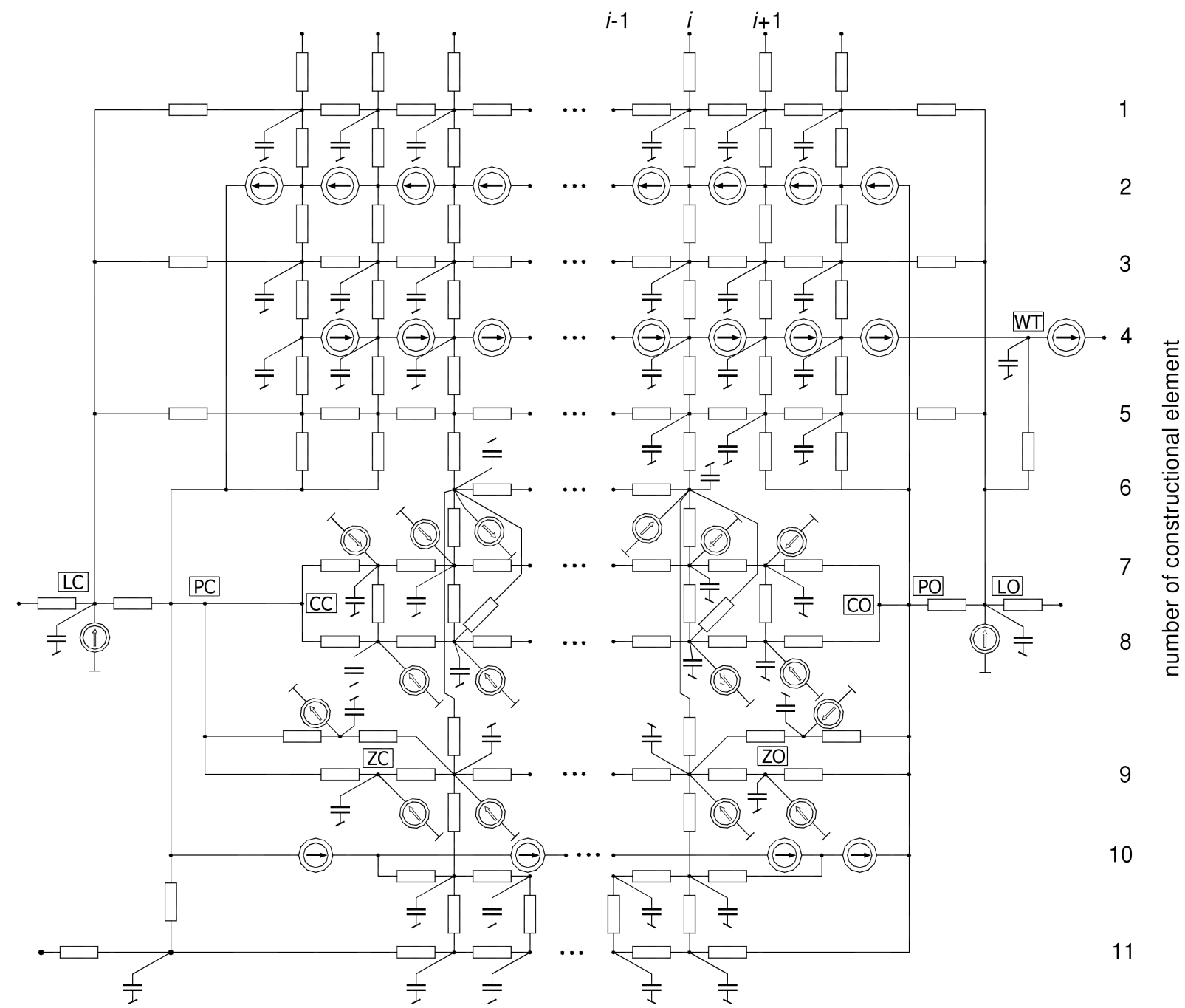

Fig. 5. Thermal network for the $85 / 250 \mathrm{~kW}$ double speed mine motor

- power losses in the stator winding and rotor cage dependent on temperature,

- power losses in the stator core and bearings practically independent of temperature,

- carrying the heat away from constructional element surfaces in the motor interior to the air streams flowing around them and from the frame external surfaces to the environment.

The thermal network for the considered $85 / 250 \mathrm{~kW}$ double speed motor contains 177 nodes. It is the result of galvanic connection of the elementary equivalent diagrams assigned to the areas separated inside the constructional elements (of structure shown in Fig. 1) and the cooling ducts (of structure shown in Fig. 2).

\section{Thermal calculations of the mine motor when designing the protection system}

In order to localize in the $85 / 250 \mathrm{~kW}$ two-speed mine motor rotor windings the places in which the maximum temperature occurs, there was calculated the temperature field in the steady thermal state corresponding to the rated load and supply conditions of the motor. The thermal calculations were performed by means of the created thermal network (Fig. 5).

Figure 6 shows the calculated axial temperature distribution in the motor stator windings for supplying the winding of the number of poles $2 p=4$ with the rated voltage and loading the motor with the rated power under steady thermal condition. The maximum temperature occurs in the middle part of the head windings of the supplied stator winding at the side $(\mathrm{O})$, so at this place the first temperature measuring sensor contained in the system of motor thermal protections should be built in. The temperature of the stator head windings at the side $(\mathrm{O})$ is higher than that at the side $(\mathrm{C})$. The water flowing in the helical duct in the motor frame is heated (Fig. 7), which causes that the temperatures of the elements at the side $(\mathrm{O})$ are higher than these of the corresponding elements at the side $(\mathrm{C})$ - it refers to the stator head windings as well

Figure 8 shows the calculated axial temperature distribution in the motor stator windings for supplying the winding of the number of poles $2 p=12$ with the rated voltage and 
loading the motor with the rated power under a steady thermal condition. The maximum temperature increase occurs in the middle part of the head windings of the supplied stator winding at the side $(\mathrm{O})$, so at this place the second temperature measuring sensor should be built in.

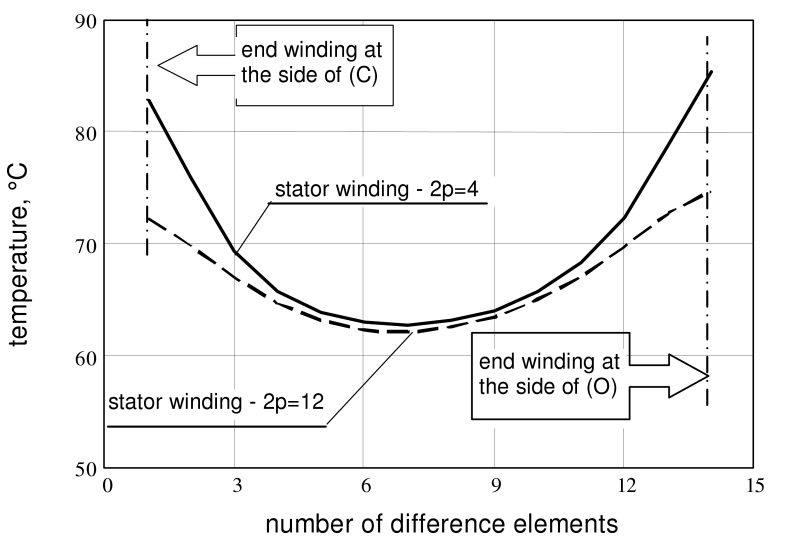

Fig. 6. Axial temperature distributions in stator windings when supplying the winding of the number of poles $2 p=4$ with the rated voltage and loading the motor with the rated power under steady thermal condition

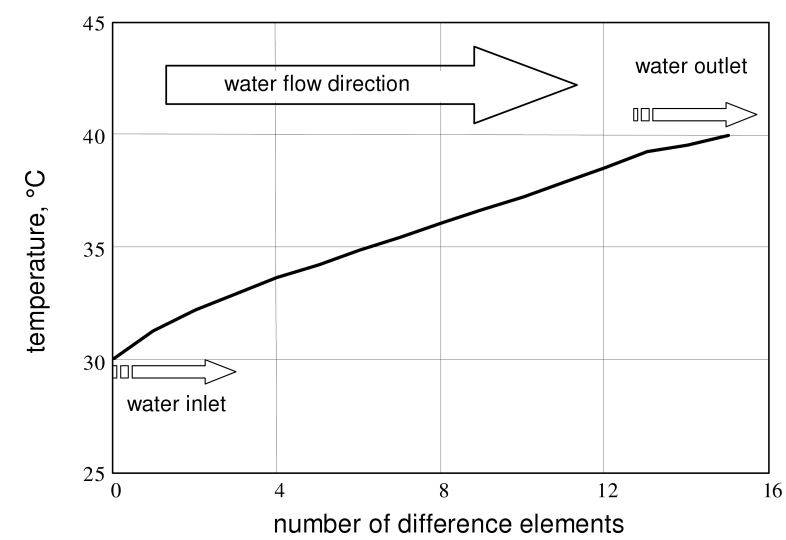

Fig. 7. Axial temperature distribution in the cooling water stream in the frame when supplying the stator winding of the number of poles $2 p=4$ with the rated voltage and loading the motor with the rated power under steady thermal condition

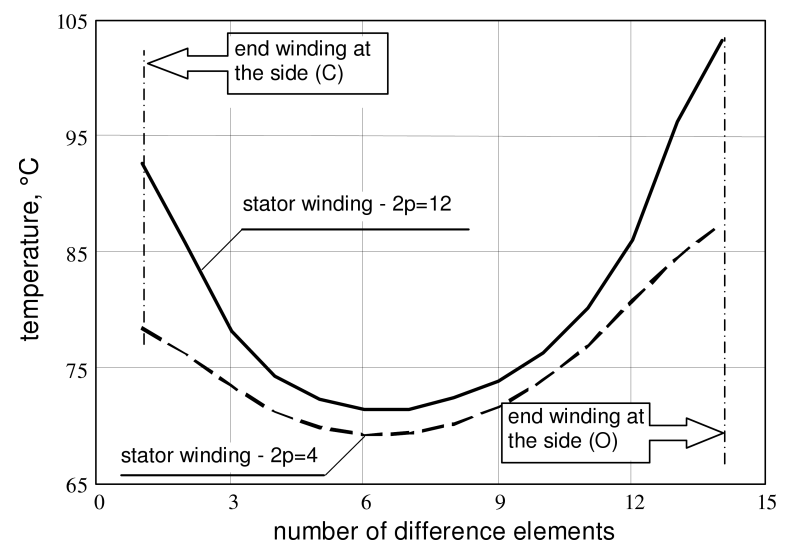

Fig. 8. Axial temperature distributions in the stator windings when supplying the winding of the number of poles $2 p=12$ with the rated voltage and loading the motor with the rated power under steady thermal condition
Additionally, there were performed calculations of the temperature field in the mine motor under steady thermal condition for:

- different cooling water discharge,

- changes of the cooling water temperature at the inlet to the frame,

- worsening the conditions of carrying away the heat from the frame due to covering the motor with coal dust.

In each of the considered operating conditions of the motor the temperatures of its elements are different. However, the maximum temperature always occurs at the same places of the stator windings, in which location of the temperature measuring sensors was proposed.

In order to determine the values of the equivalent thermal time constants of heating the stator windings, there was calculated the temperature field in the motor during a heating test under transient thermal condition. The motor was supplied with the rated voltage and loaded with the rated power. All elements of the motor as well as the cooling water and air had the same initial temperature equal to $20^{\circ} \mathrm{C}$ within their whole volume.

The calculated waveforms of the temperature in the motor stator windings during the heating test are shown in the following figures:

- Figures 9 and 10 - for supplying the winding of the number of poles $2 p=12$,

- Figures 11 and 12 - for supplying the winding of the number of poles $2 p=4$.

The equivalent thermal time constants of heating determined basing on simulations of the motor heating test are equal to:

- $T_{1}=44 \mathrm{~min}$. for the number of poles $2 p=12$,

- $T_{2}=62 \mathrm{~min}$. for the number of poles $2 p=4$.

The values of these time constants are needed when setting the microprocessor thermal protection of a mine motor.

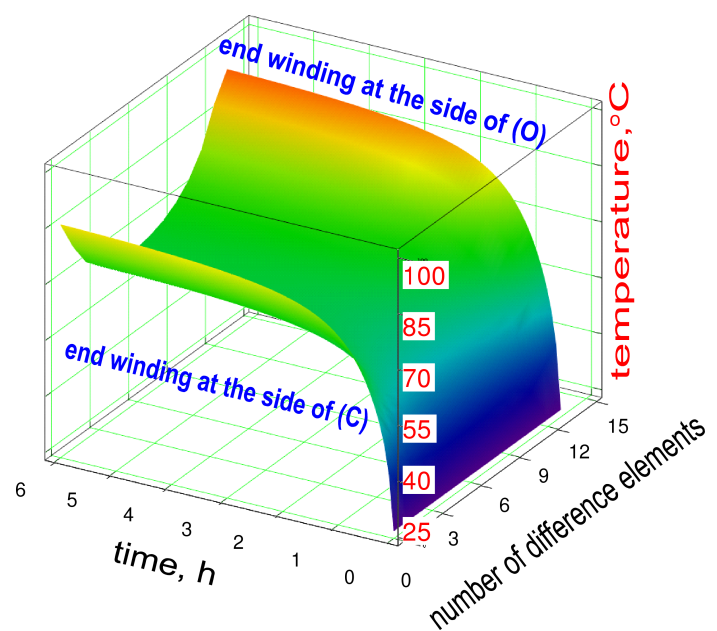

Fig. 9. Temperature distribution in the stator windings when supplying the winding of the number of poles $2 p=12$ with the rated voltage and loading the motor with the rated power $P_{n(2 p=12)}=85 \mathrm{~kW}$ during the heating test under transient thermal condition 


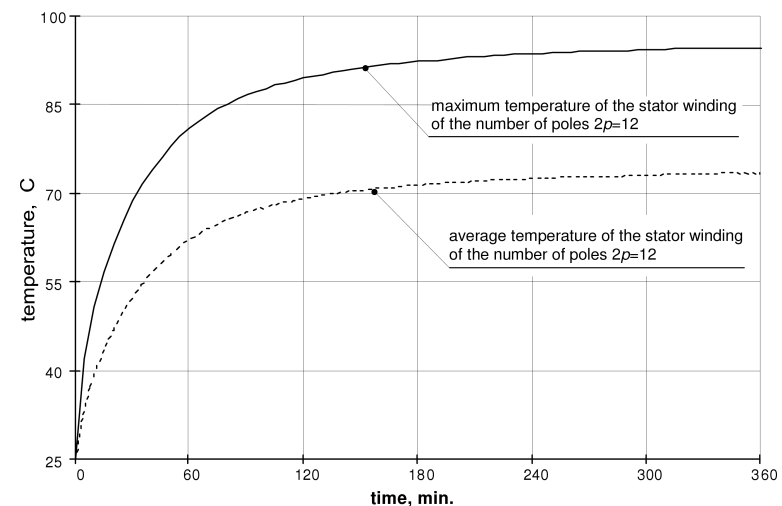

Fig. 10. Waveforms of the maximum and average temperature of the stator winding of the number of poles $2 p=12$ supplied with the rated voltage when loading the motor with the rated power $P_{n(2 p=12)}=85 \mathrm{~kW}$ during the heating test under transient thermal condition

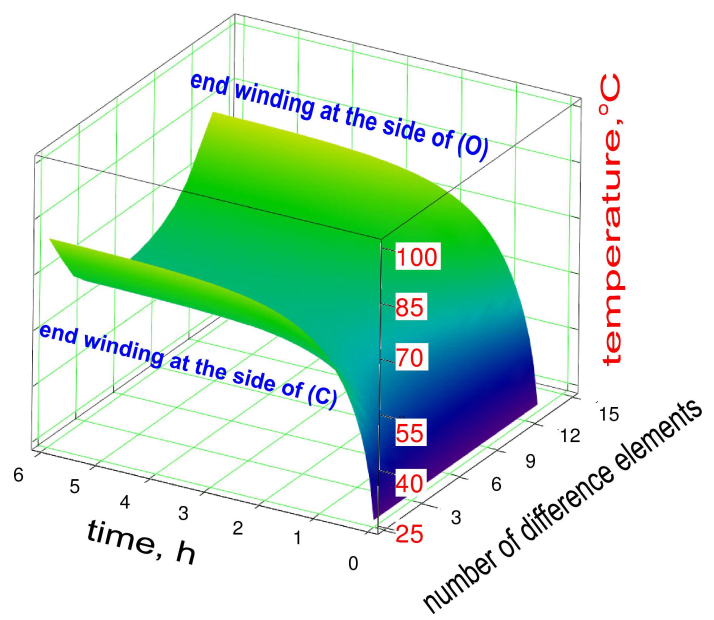

Fig. 11. Temperature distributions in the stator windings when supplying the winding of the number of poles $2 p=4$ with the rated voltage and loading the motor with the rated power $P_{n(2 p=4)}=250 \mathrm{~kW}$ during the heating test under transient thermal condition

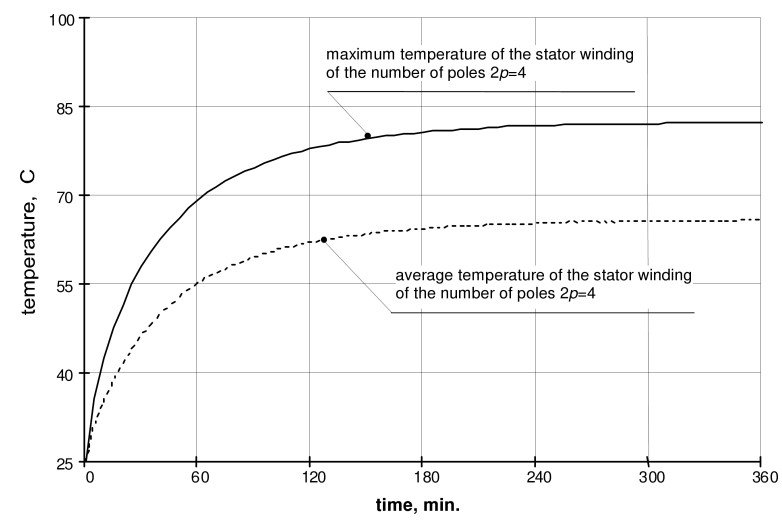

Fig. 12. Waveforms of the maximum and average temperature of the stator winding of the number of poles $2 p=4$ supplied with the rated voltage when loading the motor with the rated power $P_{n(2 p=12)}=250 \mathrm{~kW}$ during the heating test under transient thermal condition

\section{Summary}

The modified thermal networks created for mine motors cooled with water are especially helpful when designing and setting microprocessor protections cooperating with temperature measuring sensors built in the motor. They enable location of the hottest places in the motor elements endangered to thermal damage in order to built in them temperature measuring sensors. A microprocessor thermal protection with the correct values of the equivalent thermal time constants of heating the windings enables motor work at short overloads, turning it off only then, when the winding temperature reaches the permissible value.

Computer programs for calculations of the temperature field in mine motors in the steady and transient thermal states developed on a basis of the modified thermal networks are also used when designing motors and investigating hazards occurring in motors during exploitation under different environmental conditions existing in coal mine undergrounds [14], for instance increased ambient or cooling water temperature, decreased cooling water discharge, covering the motor casing with coal dust.

\section{REFERENCES}

[1] M. Ilar and T. Kley, "Multifunktionaler Motorschutz mit Relais vom Typ MCX913", Brown Boveri Mitteilungen 9, CD-ROM (1986).

[2] J. Kapinos and R. Krok, "Use of SEPAM protectionmeasurement devices manufactured by Schneider Electric for protection of induction motors", Problem Notebooks - Electrical Machines BOBRME 61, 77-81 (2000), (in Polish).

[3] J. Hak, "Die inneren axialen Wärmewiderstände einer elektrischen Maschine", Archiv für Elektrotechnik 1, 58-76 (1957).

[4] A. Cioska, B. Drak, K. Kluszczyński, R. Miksiewicz, and A. Różycki, "Computer design of asynchronous three-phase motors", Publications of Institute of Electric Machines and Apparatus of the Silesian University of Technology 1, CD-ROM (1990), (in Polish).

[5] G. Kalander, "Temperature simulation of a $15 \mathrm{~kW}$ induction machine operating at variable speed", Int. Conf. on Electrical Machines 1, 943-947 (1992).

[6] M. Rioul, "Development of thermohydraulic modelling for the determination of hot spots in the bars and the slot thermal image for the stator $900 \mathrm{MW}$ turbogenerators", Proc. ICEM'94 1, 437-441 (1994).

[7] M. Elleuch and M. Poloujadoff, "A contribution to the modelling of three phase transformers using reluctances", IEEE Trans. on Magnetics 32, 335-343 (1996).

[8] J. Mukosiej, "Universal program for thermal calculation of electric machines by the method of equivalent thermal networks (ETN)", Proc. ICEM'96 1, 377-381 (1996).

[9] D.J. Tylavsky, Qing He, Jennie Si, G.A. McCulla, and J.R. Hunt, "Transformer top - oil temperature modeling and simulation", IEEE Trans. on Industry Applications 36 (5), 1219-1225 (2000).

[10] G. Swift, T.S. Molinski, and W. Lehn, "A fundamental approach to transformer thermal modeling - Part I: Theory and 
equivalent circuit”, IEEE Trans. on Power Delivery 16, 171175 (2001).

[11] D. Gurazdowski and J. Zawilak, "Temperature distribution in the turbogenerator stator winding bar", Problem Notebooks Electrical Machines BOBRME 75, 177-184 (2006), (in Polish).

[12] E.I. Gurjewicz and A.G. Filin, "Temperature field in the large turbogenerator stator winding at local damages of the in- ternal water cooling system", Elektricestvo 3, 23-29 (2010), (in Russian).

[13] R. Krok, "Thermal networks for modelling the temperature field in AC electric machines", Monograph, Silesian University of Technology Publishing House, Gliwice, 2010, (in Polish).

[14] R. Krok, "Influence of work environment on thermal state of mine motors", Archives of Electrical Engineering 60 (3), 357370 (2011). 